\title{
Lactobacillus probiotic protects intestinal epithelium from radiation injury in a TLR-2/cyclo-oxygenase-2- dependent manner
}

\author{
Matthew A Ciorba, ${ }^{1}$ Terrence E Riehl, ${ }^{1}$ M Suprada Rao, ${ }^{1}$ Clara Moon, ${ }^{2}$ Xueping Ee, \\ Gerardo M Nava, ${ }^{2}$ Monica R Walker, ${ }^{2}$ Jeffrey M Marinshaw, ${ }^{1}$ \\ Thaddeus S Stappenbeck, ${ }^{2}$ William F Stenson ${ }^{1}$
}

\begin{abstract}
- Additional materials are published online only. To view these files please visit the journal online (http://gut.bmj. com/content/61/6.toc).

1 Departments of Medicine, Washington University Saint Louis, School of Medicine, Saint Louis, Missouri, USA

${ }^{2}$ Departments of Pathology and Immunology, Washington University Saint Louis, School of Medicine, Saint Louis, Missouri, USA
\end{abstract}

\section{Correspondence to}

Professor William F Stenson, Division of Gastroenterology, Washington University Saint Louis, 660 South Euclid Avenue, Box 8124, Saint Louis, M0 63110, USA; wstenson@dom.wustl.edu Matthew A Ciorba, Division of University, St. Louis, $660 \mathrm{~S}$. Euclid Ave, Box 8124, St. Louis, M0 63110, USA;

MCiorba@dom.wustl.edu

Revised 23 September 2011 Accepted 26 September 2011 Published Online First 24 October 2011 Gastroenterology, Washington

\section{ABSTRACT}

Background The small intestinal epithelium is highly sensitive to radiation and is a major site of injury during radiation therapy and environmental overexposure.

Objective To examine probiotic bacteria as potential radioprotective agents in the intestine.

Methods 8-week-old C57BL/6 wild-type or knockout mice were administered probiotic by gavage for 3 days before $12 \mathrm{~Gy}$ whole body radiation. The intestine was evaluated for cell-positional apoptosis (6 h) and crypt survival (84 h).

Results Gavage of $5 \times 10^{7}$ Lactobacillus rhamnosus GG (LGG) improved crypt survival about twofold $(p<0.01)$; the effect was observed when administered before, but not after, radiation. Conditioned medium (CM) from LGG improved crypt survival $(1.95$-fold, $p<0.01)$, and both LGG and LGG-CM reduced epithelial apoptosis particularly at the crypt base $(33 \%$ to $18 \%, p<0.01)$. LGG was detected in the distal ileal contents after the gavage cycle, but did not lead to a detectable shift in bacterial family composition. The reduction in epithelial apoptosis and improved crypt survival offered by LGG was lost in MyD88 ${ }^{-/-}$, TLR-2 $2^{-/-}$and cyclo-oxygenase$2^{-/-}(\mathrm{COX}-2)$ mice but not TLR-4 ${ }^{-/-}$mice. LGG administration did not lead to increased jejunal COX-2 mRNA or prostaglandin E2 levels or a change in number of COX-2-expressing cells. However, a location shift was observed in constitutively COX-2-expressing cells of the lamina propria from the villi to a position near the crypt base (villi to crypt ratio $80: 20$ for control and 62:38 for LGG; $p<0.001)$. Co-staining revealed these COX-2expressing small intestinal lamina propria cells to be mesenchymal stem cells.

Conclusions LGG or its CM reduce radiation-induced epithelial injury and improve crypt survival. A TLR-2/ MyD88 signalling mechanism leading to repositioning of constitutive COX-2-expressing mesenchymal stem cells to the crypt base is invoked.

\section{INTRODUCTION}

The small intestine epithelium and the bone marrow are highly sensitive to radiation and are the major sites of injury during radiation therapy. ${ }^{12}$ Diarrhoea induced by radiation of the small intestine is the limiting factor in the dosing of radiation malignancies. ${ }^{3}$ There is a need for agents that could therapy for rectal cancer and other abdominal

\section{Significance of this study}

What is already known about this subject?

- In radiotherapy for abdominal malignancy, radiation-induced diarrhoea from collateral small bowel epithelial injury is a dose-limiting side effect.

- Lipopolysaccharide and flagellin, TLR-4 and TLR5 ligands, have been demonstrated to be radioprotective in the small intestine.

- Radioprotection induced by parenteral lipopolysaccharide is cyclo-oxygenase-2 (COX-2) dependent.

\section{What are the new findings?}

- Orally administered Lactobacillus rhamnosus GG (LGG) probiotic and its conditioned medium protect the murine small intestinal epithelium from radiation injury.

- LGG-mediated radioprotection is dependent on MyD88, TLR-2 and COX-2 and occurs without significantly altering the bacterial family composition of the small intestine.

- Administration of LGG does not change COX-2 levels, but results in a repositioning of COX-2expressing mesenchymal stem cells of the lamina propria from the villi to the crypt region.

How might it impact on clinical practice in the foreseeable future?

- This study raises the possibility that LGG, other probiotic bacteria, or probiotic-derived products may be useful as a prophylactic strategy to limit intestinal injury to humans during radiation therapy.

be given before radiation therapy that would diminish radiation injury to the small intestine without decreasing the radiation sensitivity of the tumour.

Interactions between the commensal bacteria and the epithelium influence the epithelial response to injury. Signalling through TLRs affects epithelial proliferation in the dextran sulphate sodium (DSS) model of colitis, ${ }^{4-7}$ and microbial interactions affect the host's response to radiation. ${ }^{89}$ Bacterial products can affect the intestinal epithelial response to unlocked scheme, see http:// gut.bmj.com/site/about/ unlocked.xhtml online under the BMJ Journals 
radiation injury. Lipopolysaccharide (LPS), a TLR-4 ligand, is radioprotective in the mouse intestine through a mechanism that involves prostaglandin E2 $\left(\mathrm{PGE}_{2}\right)$ synthesis through cyclooxygenase-2 (COX-2). ${ }^{10}$ Administration of TLR-5 ligands, flagellin or CBL13502 (a polypeptide drug derived from salmonella flagellin) before irradiation protected both mice and monkeys from gastrointestinal (GI) and haematopoietic acute radiation syndromes. ${ }^{11-13}$

Probiotic therapies have been clinically evaluated and used as treatment for human inflammatory bowel diseases, pouchitis, irritable bowel syndrome and antibiotic-associated diarrhoea. ${ }^{14-16}$ For inflammatory bowel diseases, most probiotic studies have been largely descriptive, although recent investigations in mice describe anti-apoptotic and anti-inflammatory mechanisms for the probiotic, Lactobacillus rhamnosus GG (LGG). ${ }^{17} 18$

There have been preliminary studies of probiotic use in the prevention or treatment of radiation injury in the intestine. ${ }^{19}$ In experimental models of radiation injury, lactobacillus or a mixture of lactobacillus and bifidobacterium given to rodents before and after radiation resulted in improved histology ${ }^{20}$ and decreased endotoxaemia and sepsis. ${ }^{21}{ }^{22}$ No assessment was made of epithelial crypt survival or apoptosis, nor was any mechanism described. In humans, probiotics have been used to control diarrhoea after radiation exposure has begun, showing a trend towards benefit. ${ }^{23-25}$ Treatment with a probiotic combination of Lactobacillus acidophilus plus Bifidobacterium bifidum was compared with placebo in patients receiving radiation therapy with weekly cisplatin for cervical cancer. ${ }^{26}$ The combination of probiotics resulted in less diarrhoea. Since radiotherapy is a planned event and prophylactic therapy is feasible, we sought to determine if prophylactic probiotic treatment affects the intestinal epithelial response to radiation as assessed by apoptosis and crypt survival.

\section{METHODS AND MATERIALS}

\section{Mice}

All mice used were on the C57Bl/6 background. Wild-type (WT), TLR- $2^{-1-}$ and TLR-4 ${ }^{-1-}$ mice were purchased from Jackson Laboratories (Bar Harbour, Maine, USA) and bred in house. MyD88 $8^{-1-}$ and $\mathrm{COX}-2^{-/-}$mice are maintained in our facility as previously described. ${ }^{4}$ Experiments with WT mice were confirmed both on those purchased directly from Jackson Laboratories and those bred in house. Comparator groups were matched for age and sex, typically as littermates. Female mice were preferentially used. WT mice (phosphate-buffered saline (PBS) vs LGG treated) were tested concurrently as positive controls with all knockout mouse experiments. All mice were maintained on a $12 \mathrm{~h}$ light/dark schedule in a temperaturecontrolled specific pathogen free facility and fed standard laboratory mouse chow. Animal procedures and protocols were conducted in accordance with the institutional review board at Washington University School of Medicine. Whole body irradiation of mice was carried out in a Gammacell 40 137Cs irradiator (Atomic Energy of Canada Ltd, Chalk River, Ontario, Canada) at a dose rate of $80.7 \mathrm{cGy} / \mathrm{min}$ and a total dose of $12 \mathrm{~Gy}$.

\section{Apoptosis and crypt survival in mouse small intestine}

After 12 Gy whole body irradiation, mice were killed at $6 \mathrm{~h}$ (apoptosis studies) or $84 \mathrm{~h}$ (crypt survival studies). The entire small intestine was harvested and used to collect tissue for RNA or protein determination or histology. The proximal jejunum and distal ileum were fixed for histology in $10 \%$ formalin with PBS. A minimum of six $5 \mathrm{~mm}$ segments were taken from each mouse and

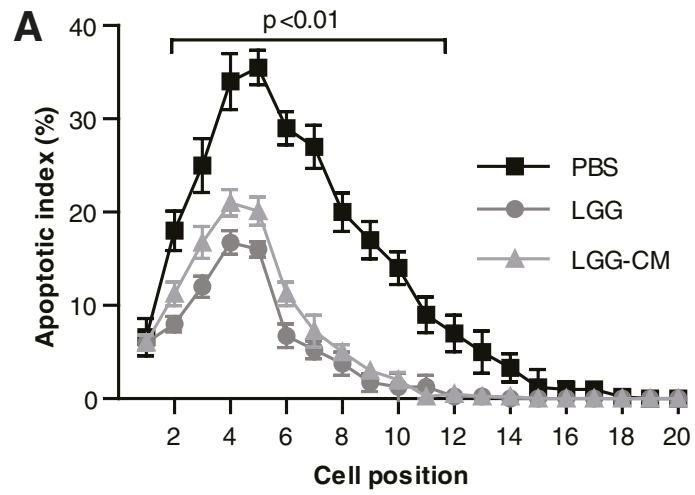

\section{Probiotics and conditioned medium}

Lactobacillus spp. were purchased from American Tissue Culture Collection (ATCC, Manassas, Virginia, USA). Lactobacillus rhamnosus GG (LGG) (ATCC 53103), Lactobacillus acidophilus 


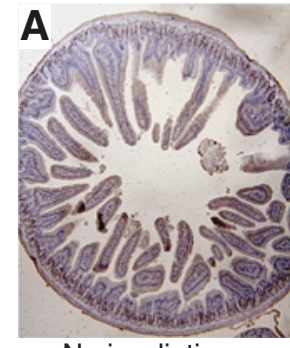

No irradiation

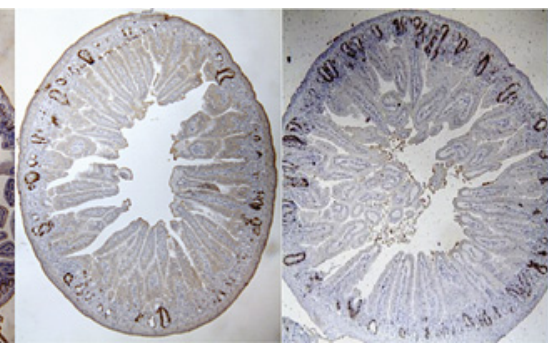

PBS

LGG pre-treatment
$84 \mathrm{~h}$ after 12 Gy IR

B
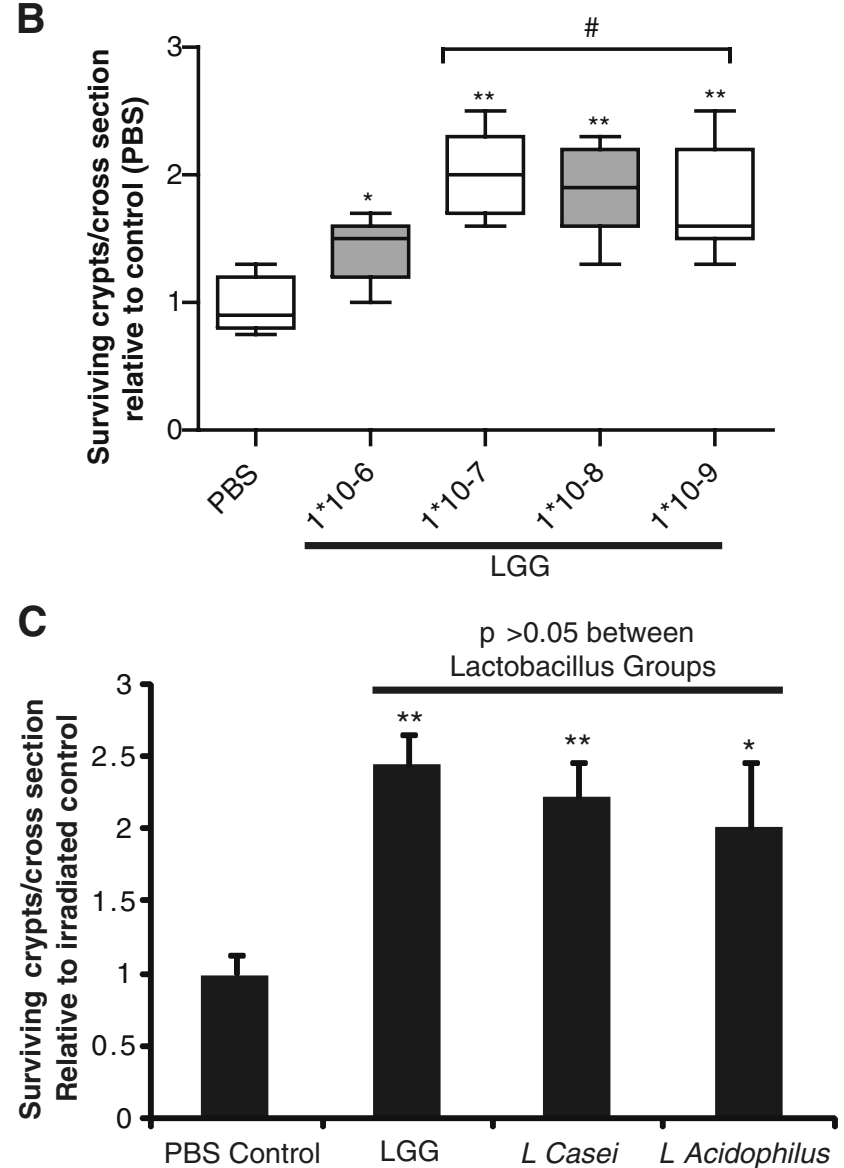

D

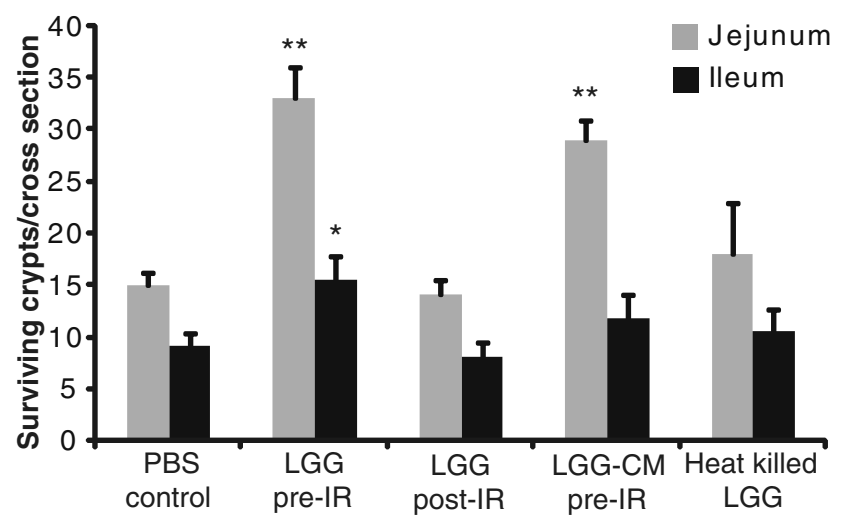

Figure 2 Crypt survival after radiation is improved by live Lactobacillus probiotics and Lactobacillus rhamnosus GG (LGG) culture medium supernatant (LGG-CM). Mice were gavaged with probiotic species described or LGG-CM for 3 days before radiation (IR) with 12 Gy. Eightyfour hours after radiation exposure, mice were injected with
(ATCC 393) and Lactobacillus casei (ATCC 4356) were cultured according to the manufacturer's recommendations in MRS broth (Fisher Scientific, Pittsburg, Pennsylvania, USA). Bacteria were cultured at $37^{\circ} \mathrm{C}$ to reach log phase growth as determined by a $0.5 A_{600}$ reading, $^{29}$ then immediately centrifuged at $1200 \times g$ for $10 \mathrm{~min}$ and washed twice with cold PBS. Bacteria were brought up in volumes to reach the desired number of bacteria per $200 \mu \mathrm{l}$ for murine gavage. Concentrations of live bacteria were confirmed by serial dilutions and plating for colony forming unit counts. Commercially marketed forms of the probiotic supplements LGG (Culturelle; Amerifit, Cromwell, Connecticut, USA) and Bifidobacterium infantis 35624 (Proctor \& Gamble, Cincinnati, Ohio, USA) were tested separately.

Heat-killed bacteria were prepared as previously described exposing LGG in PBS to boiling temperature for $30 \mathrm{~min}$ followed by a PBS wash. ${ }^{30}$ LGG conditioned culture medium (LGG-CM) was prepared by growing LGG in MRS broth to log phase growth. The cultured broth was then centrifuged $(1500 \times \mathrm{g}$ for $10 \mathrm{~min}$ ) to pellet the majority of bacteria before allowing the culture to again reach log phase growth. After a second centrifugation, the $\mathrm{CM} \mathrm{pH}$ was adjusted to that of freshly prepared control MRS broth. Both preparations were sterile filtered $(0.2 \mu \mathrm{m})$ before use.

\section{Organ-specific mesenchymal stem cells (MSCs)}

Small intestinal and colon MSCs were prepared as previously described. ${ }^{31}$ In vitro stimulation of MSCs was performed with a commercial TLR-2 ligand (Pam3CSK4, catalogue number Tlr1-pms; Invivogen, San Diego, California, USA) at the concentrations described.

\section{Protein and nucleic acid analysis}

Whole tissue RNA expression was analysed by quantitative reverse transcription (qRT)-PCR as previously described. ${ }^{32} 33$ qRT-PCR primer sequences are described in online supplementary table 1. Formalin-fixed paraffin-embedded jejunal tissues were used for immunofluorescence using high-temperature citrate-based antigen retrieval. COX-2-expressing cells were detected using a mouse monoclonal antibody (BD Biosciences, San Diego, California, USA; catalogue number 610204; diluted 1:50) followed by AlexaFluor 594 goat anti-mouse (Molecular Probes, Carlsbad, California, USA; catalogue number 11032; diluted 1:250). The surfaces of epithelial cells were labelled using rabbit anti-human $\beta$-catenin (Santa Cruz Biotechnology, Santa Cruz, California, USA; catalogue number sc-7199; diluted 1:100) followed by AlexaFluor 488 donkey anti-rabbit (Molecular Probes; catalogue number 21206; diluted 1:250). Photomicrographs were taken at $200 \times$ magnification and used to obtain counts of COX-2-expressing stromal cells in the crypt and villus

\footnotetext{
(Continued)

bromodeoxyuridine (BrdU). Intestinal tissues were harvested 90 min later and fixed for BrdU staining. (A) A representative jejunal crosssection from an untreated mouse is compared with sections from mice exposed to radiation which received pretreatment with either phosphatebuffered saline (PBS) (control) or LGG. (B) Dose finding comparison showed no additional benefit at bacterial loads over $1 \times 10^{8}$, with a dose of $5 \times 10^{7}$ being used for subsequent experiments. ${ }^{*} p<0.05$ and ${ }^{* *} \mathrm{p}<0.01$ vs PBS; \# no significant difference between groups. (C) Three different Lactobacillus spp. were evaluated and the mean gain in jejunal regenerative crypts is shown. ${ }^{*} p<0.05$ and ${ }^{* *} p<0.01$ vs PBS. (D) The number of regenerative crypts for each listed treatment group is shown for the proximal jejunum and distal ileum (mean $+S E M, n \geq 8$ mice/group, $\geq 2$ experiments). ${ }^{*} p<0.05$ and ${ }^{* *} p<0.01$ vs PBS.
} 
Table 1 Crypt survival after pretreatment with probiotics

\begin{tabular}{llr}
\hline Treatment & Crypt survival & p Value \\
\hline PBS control & $1.00 \pm 0.15$ & \\
LGG (cultured/washed) & $1.98 \pm 0.23$ & $<0.01$ \\
Culturelle & $1.64 \pm 0.13$ & 0.01 \\
Bifidobacterium infantis 35624 & $1.09 \pm 0.18$ & 0.37
\end{tabular}

\begin{abstract}
Mice were given probiotics (LGG at $5 \times 10^{8}$ bacteria, Culturelle and $B$ infantis at 1:500th capsule) for 3 days before irradiation with $12 \mathrm{~Gy}$ and killed at $84 \mathrm{~h}$ after irradiation. Jejunal crypt survival was assessed by bromodeoxyuridine incorporation. Mean \pm SEM crypt survival is presented as normalised to PBS control. $p$ Value is compared with PBS control.
\end{abstract}

LGG, Lactobacillus rhamnosus GG; PBS, phosphate-buffered saline.

regions. At this magnification, each picture covered a length of $340 \mu \mathrm{m}$. Data are the mean number of cells per picture $(340 \mu \mathrm{m}$ length) for 20 locations along the lengths of the jejunal tissue sections from five mice. This encompassed >800 COX-2expressing cells in more than $120 \mathrm{crypt} /$ villus structures. The $\mathrm{PGE}_{2}$ assay was performed as previously described ${ }^{33}$ using an ELISA (Cayman Chemical, Ann Arbor, Michigan, USA; catalogue number 514010) according to the manufacturer's protocol. Flow cytometry was also performed as previously described with identical antibodies. ${ }^{31}$

To evaluate the effect that LGG gavage has on small intestinal bacterial composition, we used a genomic DNA-based approach as previously described. ${ }^{34}$ A complete methodological description is available in supplementary information online. Briefly, ileal contents and mucosal scrapings were obtained from groups of mice $12 \mathrm{~h}$ after the last of three gavages with PBS or live washed LGG in PBS. Bacterial DNA concentrations were estimated by quantitative PCR (qPCR) assays using validated primers targeting total bacteria (rpoB) and family-specific primers targeting $16 \mathrm{~S}$ rRNA. $^{35}$ For quantification of LGG, a qPCR assay was standardised using validated strain-specific primers. $^{36} 37$

\section{Statistical analysis}

The two-sided, non-paired Student $t$ test was used for most comparisons between treatment and control groups including crypt survival, positional apoptosis, bacterial concentrations, and weight loss by day. Survival data were assessed using the Mantel-Cox log rank test. Data are expressed as mean \pm SEM unless otherwise noted. Prism GraphPad Software (LaJolla, California, USA) was used for data analysis and graph generation.

\section{RESULTS}

\section{LGG and LGG-CM diminish radiation-induced apoptosis in a position-dependent fashion}

In WT mice, 12 Gy of radiation induced small intestinal epithelial cell apoptosis in a position-dependent fashion (figure 1A). Assessment of apoptosis by H\&E or TUNEL assay gave equal results (figure $1 \mathrm{~B}$ ). Six hours after irradiation, the apoptotic index was $>30 \%$ in positions $4-5$ and fell off gradually to below $10 \%$ at position 12 . Mice pretreated with LGG or LGG-CM showed a significant reduction in position-dependent apoptosis. LGG and LGG-CM reduced apoptosis at position 4 from $33 \%$ to $17 \%$ and $20 \%$, respectively. Position 4 is the position of the purported quiescent epithelial stem cell. ${ }^{38}$ The effects of LGG and LGG-CM in the proximal jejunum were similar. There was no significant difference in the apoptotic index between control groups gavaged with $\mathrm{PBS}$ or uncultured MRS medium (data for MRS not shown). The radioprotection of LGG or LGG-CM was apparent both in mice purchased from a commercial vendor and those with lifetime exposure to our institutional housing facility.

\section{Lactobacillus probiotics and LGG-CM enhance intestinal crypt survival}

In the absence of radiation, there were about 120 crypts per cross-jejunal section. Eighty-four hours after a single dose of 12 Gy radiation in WT mice there averaged 15 surviving crypts per cross-section. Pretreatment with LGG increased the number of surviving crypts approximately twofold (figure 2A). In dose-response studies, LGG offered some protection at a dose of $1 \times 10^{6}$ bacteria per treatment, with improved efficacy at doses of $\geq 1 \times 10^{7}$ bacteria per treatment (figure $2 \mathrm{~B}$ ). Pretreatment with $L$ casei and $L$ acidophilus also increased crypt survival (figure $2 \mathrm{C}$ ). Observed crypt survival was highest in the LGG group, although the difference between Lactobacillus spp. was not significant.

Pretreatment with LGG significantly increased the number of surviving crypts along the length of the small intestine, both in the proximal jejunum and distal ileum (figure 2D). In contrast, the administration of LGG after irradiation had no effect on the number of surviving crypts. Pretreatment with LGG supernatant increased the number of surviving crypts from 15 to 29. In contrast with the increase in crypt survival with live LGG or with LGG-CM, there was no significant increase in crypt survival with heat-killed LGG. To determine if the effects of the LGG supernatant could be active systemically as well as topically, LGG-CM was administered at equal dosing intraperitoneally to mice before radiation. Although mice tolerated this injection without adverse outcome, no benefit to crypt survival was observed (data not shown).

Administration of a commercially available LGG product (Culturelle; 1/500th of a capsule dissolved in PBS) resulted in a level of radioprotection similar to that produced by live cultured LGG and significantly greater than PBS control (table 1). This benefit was not offered with equal dosing of a nonlactobacillus-containing commercially available probiotic $(B$ infantis 35624; Align), suggesting that Lactobacillus may be the favoured probiotic genus for limiting radiation-induced small intestinal injury.

\section{LGG before radiation improves survival, diminishes weight loss} and reduces intestinal crypt loss

Small intestinal epithelial damage and weight loss are components of the radiation GI syndrome. Irradiated mice consistently lost weight after irradiation, reaching $70 \%$ of their starting weight by 7 days (figure 3A). Mice pretreated with LGG lost weight for 5 days after irradiation and then began to regain their lost weight before reaching a plateau and ultimately losing weight again. In mice, death is a standard end point measured in the study of acute GI radiation syndrome and typically occurs within the first 10 days after exposure. ${ }^{1}$ In our experiments, irradiated control mice began to die at 6 days after irradiation and mortality was $100 \%$ by day 11 (figure 3B). In contrast, mice pretreated with LGG began to die at 10 days after irradiation and some survived to day 14. Death after day 10 is described as most attributable to the combined effects of GI toxicity and bone marrow failure. Histological examination at day 7, when the greatest separation in weight curves was observed, revealed a larger number of villi in LGG-pretreated mice than in irradiated controls (figure $3 \mathrm{C}, \mathrm{D}$ ). 
A

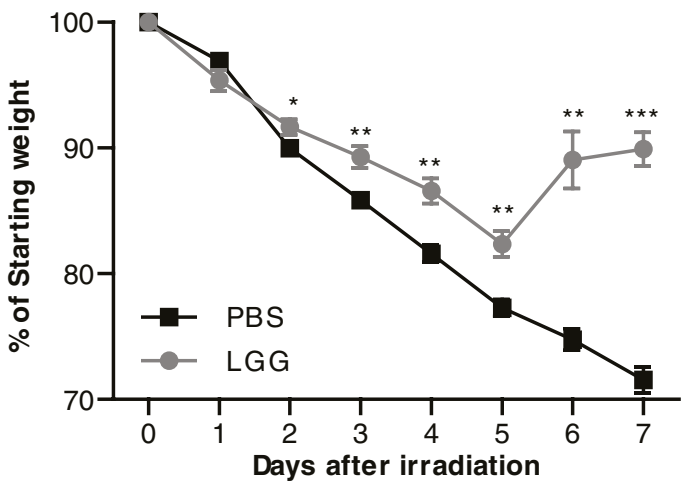

B

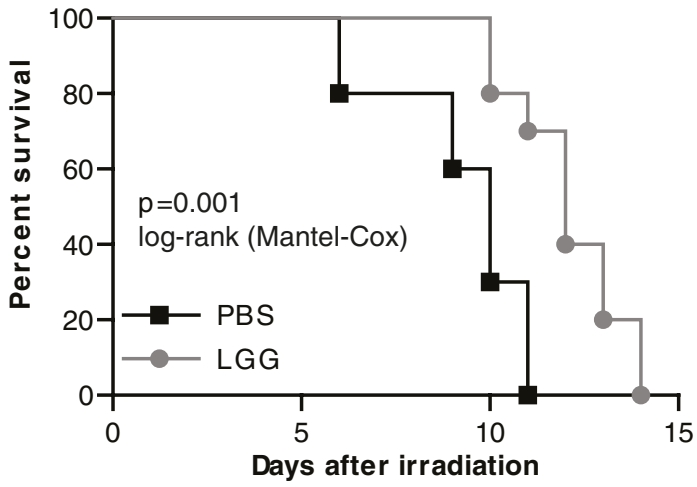

C

PBS

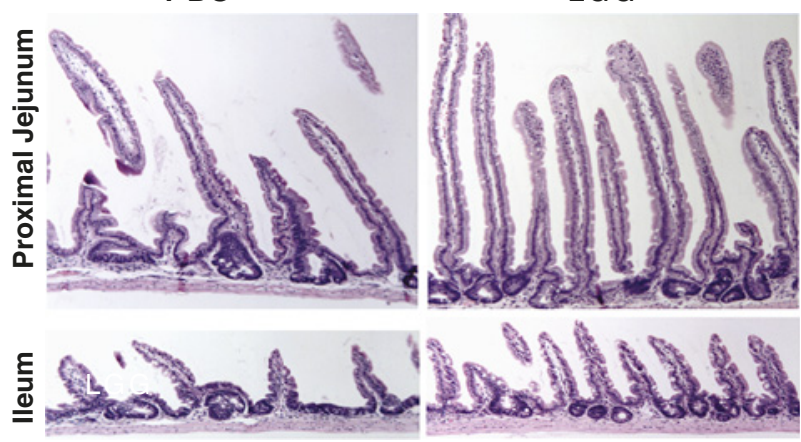

D
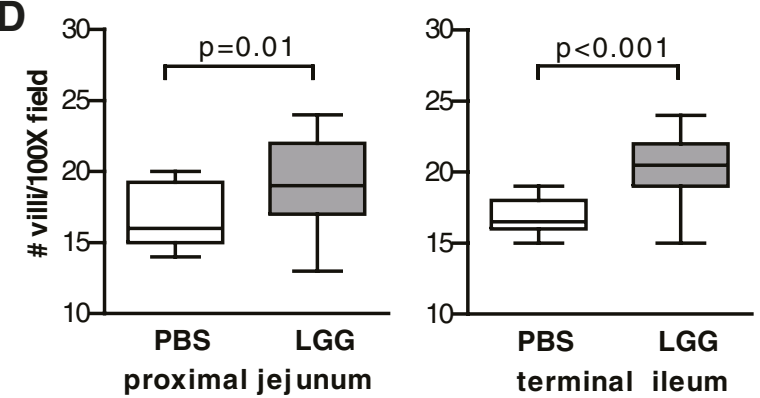

Figure 3 Lactobacillus rhamnosus GG (LGG) pretreatment improves outcomes of radiation-induced gastrointestinal syndrome. Mice received 3 days of oral gavage with phosphate-buffered saline (PBS) or LGG before $12 \mathrm{~Gy}$ whole body irradiation, after which no further treatment was given. Mice were followed for weight loss $(A)$ and survival $(B)$. Intestinal tissues harvested from a similar experiment at 7 days were fixed as longitudinal sections and evaluated for histological changes. Representative images from the proximal jejunum and terminal ileum are shown at $200 \times(C)$. The number of intact villi were counted from on four fields $(100 \times)$ from each mouse and are shown for both the ileum and jejunum (D). Weight loss and villi counts, $n=5 /$ group in each of two separate experiments. Survival, $n=10 /$ group in each of two separate experiments. ${ }^{*} p<0.05,{ }^{* *} p<0.01,{ }^{* *} p<0.001$ vs PBS.

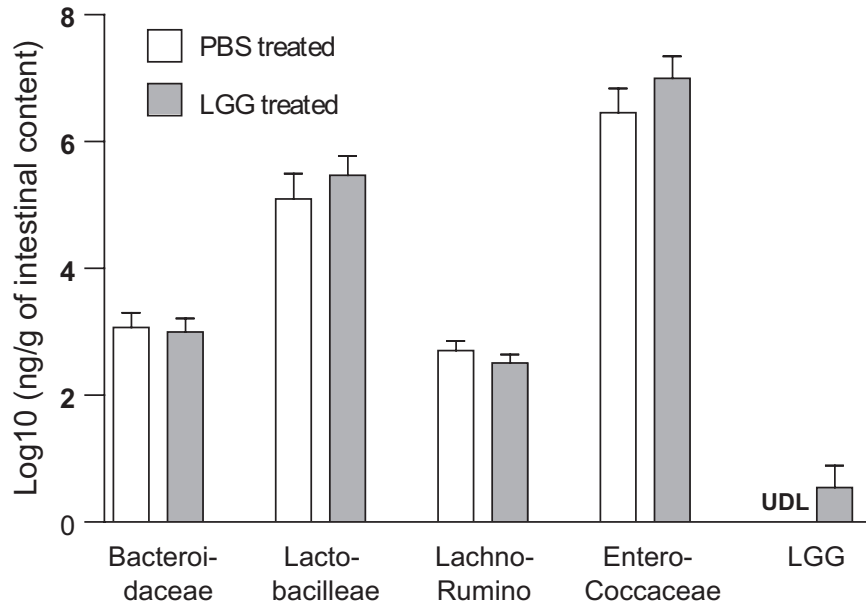

Figure 4 Effect of Lactobacillus rhamnosus GG (LGG) oral inoculation on bacterial families in the mouse ileum. Mice were gavaged with phosphate-buffered saline (PBS) or LGG for three consecutive days and killed at $12 \mathrm{~h}$ after the last gavage. Bacterial densities of distal ileal intestinal contents with mucosal scrapings were examined using LGG and family-specific quantitative PCR (qPCR) assays. Data depict relative density of each bacterial family as measured by qPCR and DNA standard curves. Data were log10 normalised before statistical analysis was performed. No statistically significant changes were noted in bacterial families ( $>>0.05$ ) after LGG treatment. $N=7$ per group. LGG was detected in $0 \%$ of PBS-treated mice and $43 \%$ of LGG-treated mice. Lachno-Rumino, Lachnospiraceae-Ruminococcaceae; UDL, under detection limit (1.38 pg DNA/ $\mu$ l).

\section{LGG treatment and small intestinal microbial composition}

Since change in commensal bacteria composition is a potential mechanism by which probiotics exert their effect, we examined the impact of LGG gavage on small intestinal microbial composition using a genomic-based approach. As the biological end point of improved epithelial crypt survival was identified in the ileum, this intestinal segment was analysed using the luminal contents and mucosal scrapings of mice treated with PBS or LGG. No significant changes were found in total bacteria (rpoB gene copy 2.86 (PBS treated) vs 2.84 (LGG treated) $\log 10 \mathrm{ng} / \mathrm{g}$ intestinal sample, $\mathrm{p}>0.05)$, nor was a shift in bacterial family composition found (figure 4). In the LGG treatment group, a detectable level of LGG was found in three of seven mouse ileums at $12 \mathrm{~h}$ after the third daily gavage.

The increased crypt survival seen with the administration of LGG is dependent on TLR-2, MyD88 and COX-2 signalling

Having found that administration of LGG to WT mice results in diminished radiation-induced apoptosis and increased crypt survival, we next sought to define a signalling pathway for these effects. Mice lacking MyD88 signalling exhibited similar baseline apoptosis to WT mice, but modestly higher crypt survival. Administration of LGG or LGG-CM to MyD88 ${ }^{-1-}$ mice had no effect on either crypt survival or apoptosis (figure 5A,B). This suggests that the radioprotective effects of LGG in WT mice are mediated through a MyD88-dependent mechanism. Lactobacilli are Gram-positive bacteria exhibiting a cell well containing lipoteichoic acid, but lacking flagella or LPS. Thus we chose to investigate whether TLR-2 was involved in Lactobacillus-mediated radioprotection. In TLR-2 ${ }^{-/-}$mice, prophylactic LGG treatment offered no reduction in epithelial cell positional apoptosis or improvement in crypt survival, suggesting that the radioprotective effects of LGG in WT mice are mediated through 

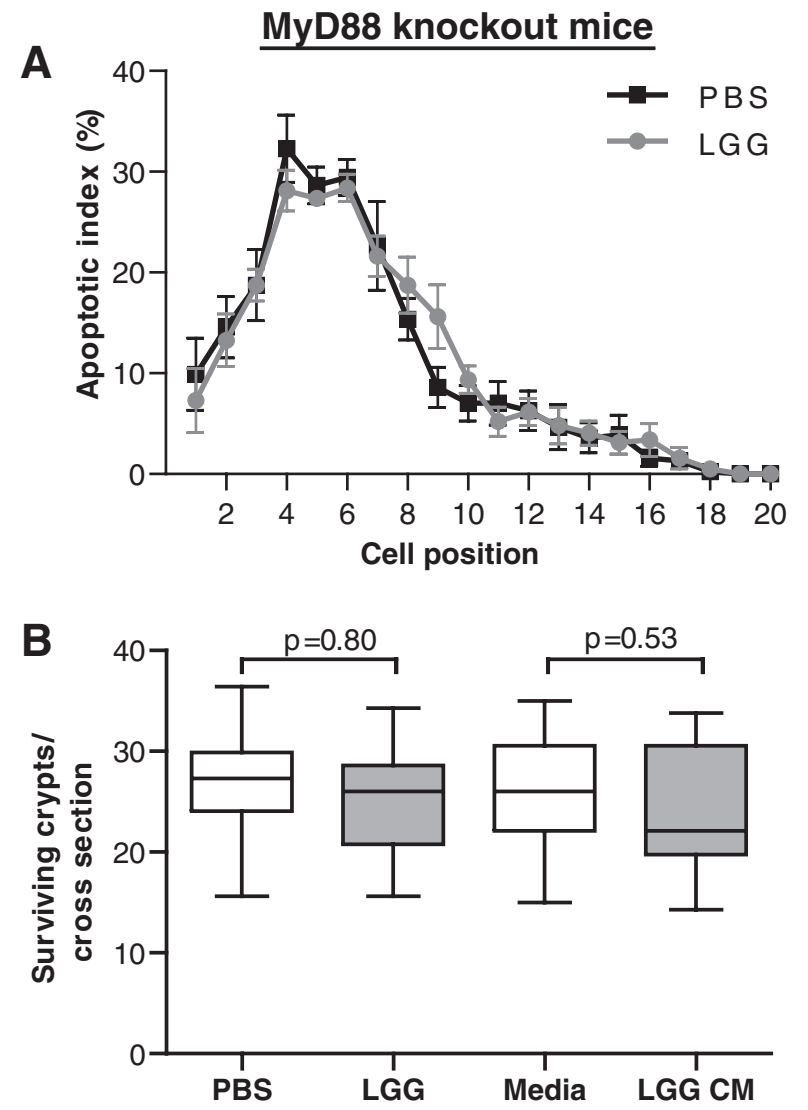

TLR-2 knockout mice

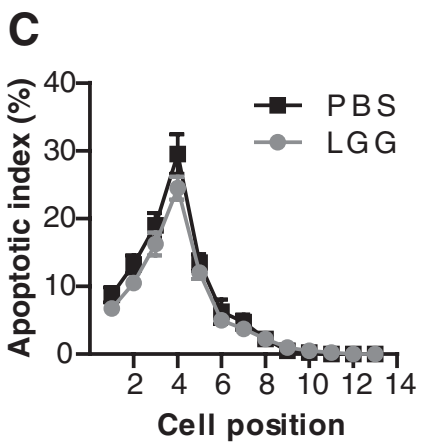

D

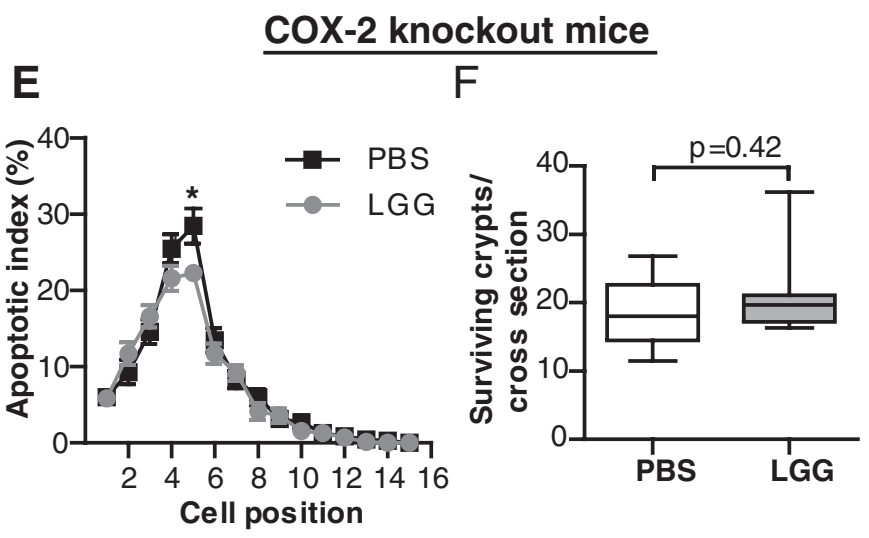

Figure 5 Radioprotective effects of Lactobacillus rhamnosus GG (LGG) require TLR-2/MyD88 signalling and cyclo-oxygenase 2 (COX-2). (A) In

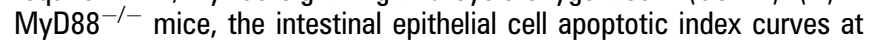
$6 \mathrm{~h}$ showed no significant difference between mice treated with phosphate-buffered saline (PBS) or live LGG for 3 days before radiation
TLR-2 (figure 5C,D). As a negative control, we examined the specificity of the TLR-2/MyD88 pathway by confirming the presence of LGG-mediated intestinal radioprotection in TLR-4 $4^{-/}$mice (mean crypt survival/jejunal cross-section $13.2 \pm 2.1$ vs $22.6 \pm 3.1$ for PBS vs LGG; $\mathrm{p}=0.02$ ). We suspected that TLR-4 signalling would not play a role in the radioprotective effects of LGG because LGG is a Gram-positive organism and does not make LPS.

We have previously demonstrated that the radioprotective effects of LPS are mediated through the induction of COX-2 expression and the synthesis of $\mathrm{PGE}_{2}{ }^{10}$ We sought to determine if this pathway is involved in the radioprotective effects of LGG. At baseline, radiation-induced apoptosis in mice with disrupted COX-2 was modestly diminished compared with WT mice (figure $5 \mathrm{E}$ ). The administration of LGG to $\mathrm{COX}-2^{-/-}$mice resulted in a small but significant further decrease in apoptosis only at position 5 (from $28 \%$ to $22 \%$ ), a reduction less striking than that seen in the WT mice. Baseline crypt survival after radiation in $\mathrm{COX}-2^{-/-}$mice was similar to that seen in WT mice, and administration of LGG to $\mathrm{COX}-2^{-1-}$ mice did not improve crypt survival (figure $5 \mathrm{~F}$ ). These results suggest that the radioprotective effects of LGG on crypt survival are mediated at least in part through COX-2.

Having found that signalling through COX-2 is important to the radioprotective effects of LGG, we next sought to determine if administration of LGG resulted in an increase in COX-2 expression. Administration of LGG by gavage did not result in increases in intestinal COX-2 mRNA or intestinal $\mathrm{PGE}_{2}$ levels (figure 6A,B). We previously demonstrated that in DSS-induced colitis, COX-2-expressing MSCs in the distal colon migrate from positions in the lamina propria that are adjacent to the upper parts of the crypts to positions in the lamina propria adjacent to the more rapidly proliferating epithelial cells in the lower crypts. ${ }^{4}$ In light of this observation, we sought to determine if the administration of LGG resulted in the migration of COX-2expressing cells in the small intestine. Since the effect of LGG was prophylactic in preventing injury, we examined unirradiated mice for this shift. The LGG dosing schedule used here was the same as that used to demonstrate radioprotection. While there was no difference in the number of COX-2-expressing stromal cells after LGG, there was a shift in cell localisation from the villi to the crypt region (figure 6C,D). In PBS-gavaged mice, we found $80 \%$ of COX-2-expressing cells in the lamina propria of the villi, with $20 \%$ residing in the lamina propria surrounding the crypts. After LGG, however, $62 \%$ of the COX-2-expressing cells were associated with the villi, and $38 \%$ were in the crypt region (figure $6 \mathrm{E})$. Thus we observed a highly significant $(p<0.001)$ shift in COX-2-expressing cells from the villi to the crypt region after LGG exposure in WT mice. In TLR-2 ${ }^{-1}$ mice gavaged with LGG, no shift in COX-2-expressing cells occurred ( $p=0.34)$. At baseline, TLR-2 $2^{-/-}$mice exhibited a greater proportion of COX2 -expressing cells already in the crypt region. Overall these

\section{(Continued)}

( $n=6 /$ group). (B) MyD88 ${ }^{-/-}$mice showed no change in crypt survival after gavage with LGG or LGG conditioned medium (LGG-CM) when compared with PBS or media controls ( $\mathrm{n}>10$ total/group over two or more experiments). (C) TLR-2 ${ }^{-/}$mice exhibit similar epithelial crypt apoptosis index with LGG treatment to PBS ( $n=4 /$ group). (D) Crypt survival is not improved by LGG in TLR-2 ${ }^{-/-}$mice ( $n=9 /$ group over two separate experiments). (E) COX-2 $2^{-1-}$ mice were evaluated for apopotosis ( $n=7 /$ group) and (F) surviving crypts ( $n=9 /$ group). FSC, forward scatter. ${ }^{*} p \leq 0.05$ vs LGG. 
A

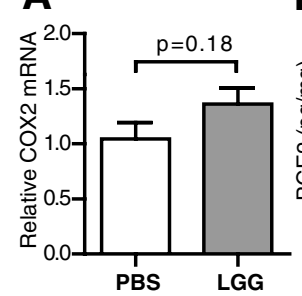

B

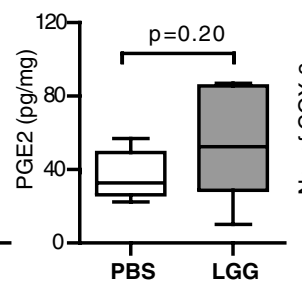

C

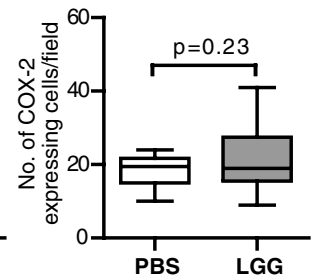

D

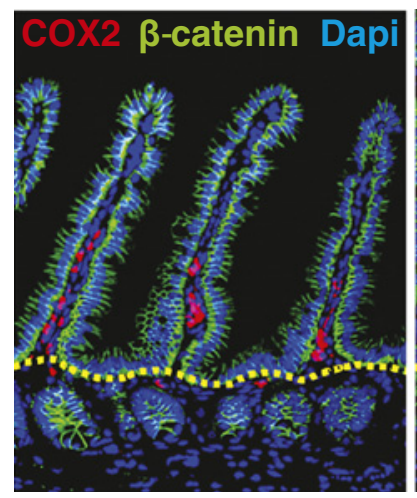

Control

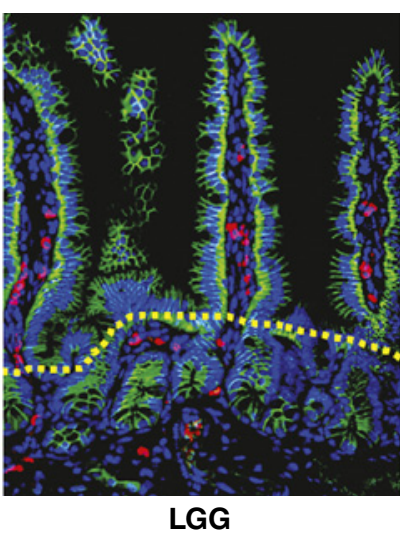

E

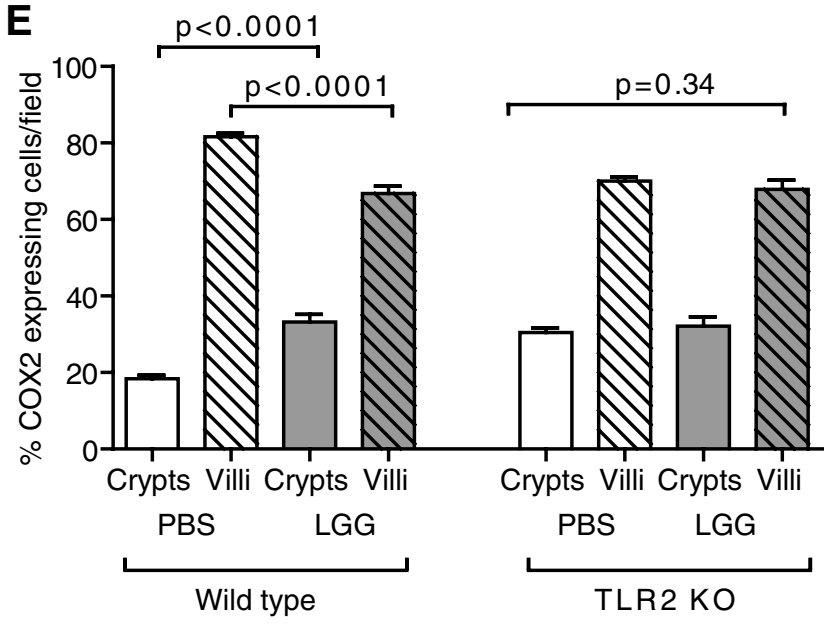

Figure 6 Lactobacillus rhamnosus GG (LGG) shifts prostaglandinproducing cells. Wild-type mice received 3 days of gavaging with phosphate-buffered saline (PBS) or LGG, and proximal to mid jejunal tissues were harvested for analysis. (A) Relative expression of cyclooxygenase 2 (COX-2) mRNA measured by quantitative reverse transcription-PCR. (B) ELISA analysis of prostaglandin $2\left(\mathrm{PGE}_{2}\right)$ production. (C) Total number of COX-2-expressing cells per $200 \times$ field. (D) Immunofluorescence showing COX-2-expressing cells within the crypt/villus structure. The crypt/villus junction is demarcated by a yellow dotted line $(200 \times)$. (E) Position of COX-2-positive cells in relation to crypt/villus junction expressed as percentage of total COX-2-positive cells in wild-type and TLR-2 ${ }^{-/-}$(TLR2 KO) mice. $\mathrm{N}=5$ mice per group/ experiment; experiment completed twice.

findings support the feasible link between LGG, TLR-2 signalling and COX-2-dependent radioprotection.

We next sought to further characterise the identified COX-2positive cells of the lamina propria. By immunofluorescence, most of the COX-2-positive cells were CD44 positive and many were positive for CD29 and CD106 (figure 7A). This pattern of expression is consistent with these COX-2-positive cells being MSCs. We had previously demonstrated that colonic MSCs had high levels of COX-2 expression. ${ }^{31}$ We now isolated small intestinal MSCs and found that they also had high levels of COX-2 expression (figure $7 \mathrm{~B}$ ) and by flow cytometry were positive for MSC markers CD29, CD44 and CD106, and negative for cell surface markers CD45 (haematopoietic), F4/80 (macrophage) and PECAM (endothelial) (figure 7C). qRT-PCR was used to identify TLR expression on these MSCs, with TLRs 2, 3, 4 and 5 showing the highest expression levels (figure 7D). No PCR product was observed on gel electrophoresis for murine TLR-13. In vitro exposure of the MSCs to a TLR-2 ligand resulted in increased tumour necrosis factor $\alpha$ and COX-2 mRNA, demonstrating functional relevance to the TLR-2 expression (figure 7E).

\section{DISCUSSION}

Administration of probiotic Lactobacillus spp. before irradiation results in decreased epithelial apoptosis and increased crypt survival in the mouse small intestine. In this study, the greatest protection was offered by administration of LGG, although gavage with other Lactobacillus spp. or LGG-CM also showed significant benefit. Administration of a Bifidobacterium sp. was not radioprotective in this model. The radioprotective effects mediated by LGG required intact signalling of TLR-2, MyD88 and COX-2. LGG was protective when given before, but not after, radiation, suggesting that LGG acts by preventing radiation-induced injury rather than enhancing repair.

Peptidoglycan and lipoteichoic acid, components of the cell wall of Gram-positive bacteria including LGG, are TLR-2 ligands. The finding that LGG-CM is also radioprotective suggests that LGG peptidoglycan or lipoteichoic acid is released into the medium and is capable of binding TLR-2. This is the first demonstration that a probiotic-based biological effect is mediated through TLR-2. The only previous demonstration of the beneficial effects of a probiotic being mediated through TLR signalling was the report that anti-inflammatory effects of VSL\#3, a mixture of different bacteria, are mediated through TLR-9. ${ }^{39}$ Heat shock proteins, reactive oxygen species and AKT activation have all been implicated in mediating the biological effects of LGG. ${ }^{18} 3040$ Whether TLR-2 activation is also involved in mediating these effects has not been addressed. The positional curves for radiation-induced apoptosis were different in the TLR-2 $2^{-/-}$and MyD88 ${ }^{-/-}$mice, raising the possibility that other TLRs may also play a role in the host response to radiation.

Yan et al described two novel proteins secreted by LGG, p40 and $\mathrm{p} 75$, that induce AKT activation and inhibit tumour necrosis factor-induced apoptosis in human and mouse epithelial cell lines and in mouse colonic explants. ${ }^{17} 30$ While it is possible that these proteins contribute to radioprotection, it is unlikely that one or both of these proteins is solely responsible for the decrease in radiation-induced apoptosis and increased crypt survival induced by LGG. The radioprotective effects seen with LGG are mediated by MyD88 and COX-2. The role of TLRs and COX-2 in mediating the effects of p40 and p75 has not been addressed. However, it was observed that the p40 and p75 proteins were expressed and cytokine protection was exhibited by both LGG and $L$ casei, although not by $L$ acidophilus. In contrast, $L$ acidophilus was effective at reducing radiationinduced epithelial injury in the present study. The explanation for possible genus specificity for lactobacilli in radioprotection is not known. While bifidobacteria are also Gram-positive bacteria, the species we tested was not radioprotective. LGG-CM is protective and the radioprotective effects of LGG are TLR-2 dependent. This suggests that LGG may release a TLR-2 ligand into the medium. The failure of bifidobacterium, another Grampositive bacterium, may be related to the failure to release 
A
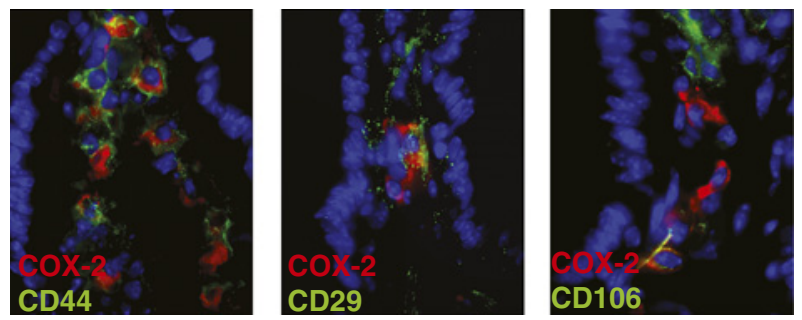

B

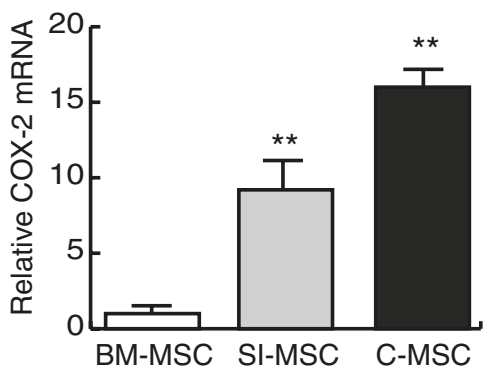

C
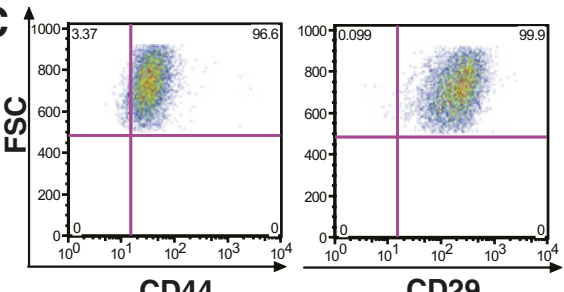

CD29

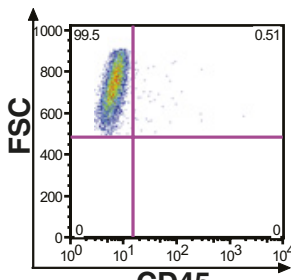

CD45

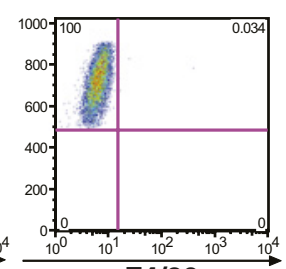

$\mathrm{F} 4 / 80$

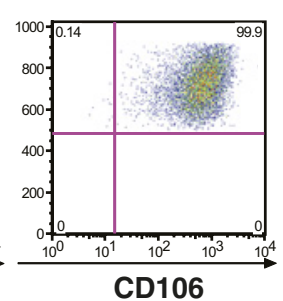

CD106

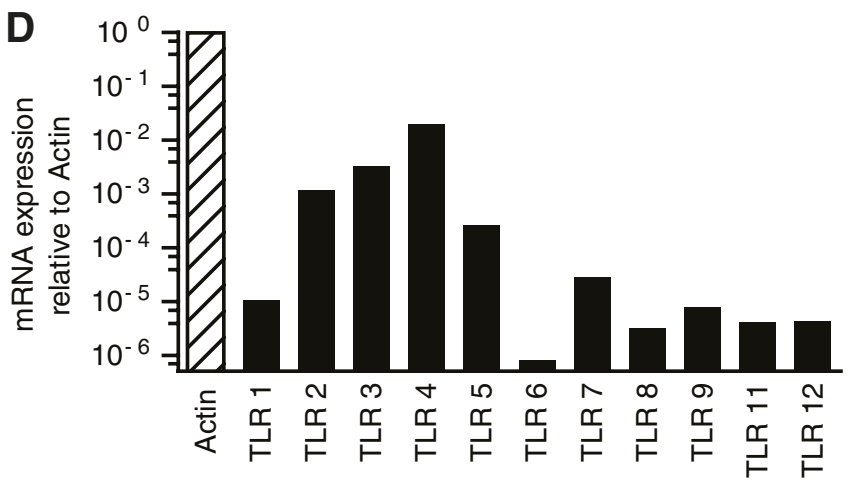

$\mathbf{E}$

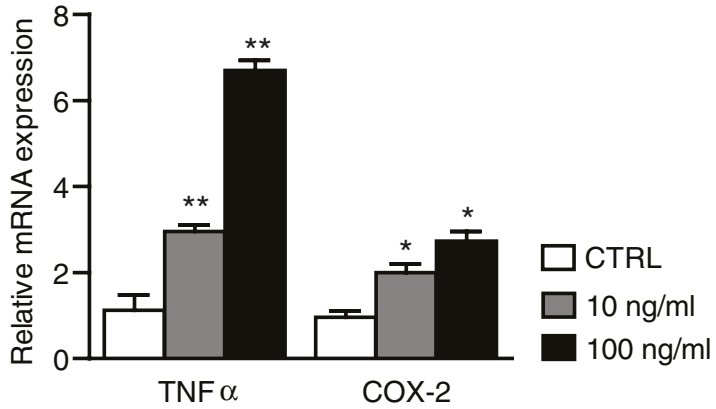

Figure 7 Characterization of small intestinal cyclo-oxygenase 2 (COX2)-positive cells. (A) Immunofluoresence co-staining of jejunal COX-2expressing cells and mesenchymal stem cell (MSC) surface markers. (B) a TLR-2 ligand, adherence properties, differential positioning of the bacterium with respect to the intestinal epithelium and the mucous layer, or differential signalling by its lipoteichoic acidbased outer membrane. ${ }^{41-43}$ The finding of differential efficacy among specific probiotic species and strains has been reported for other conditions. ${ }^{44}$ Dosage, potency and/or host handling are other possible explanations for the differences in efficacy.

Radioprotective effects of LGG are mediated through COX-2. $\mathrm{PGE}_{2}$, which can be produced through either COX-1 or COX-2, is radioprotective in the intestine. ${ }^{10}$ The radioprotective effects of LPS, acting through TLR-4, are dependent on COX-2 and $\mathrm{PGE}_{2}{ }^{10}$ Parenteral administration of LPS induces increased COX2 expression in the intestine. Although LGG also acts through a COX-2-dependent mechanism, gavage with LGG affected neither the expression of COX-2 nor the level of PGE2 in the small intestine. LGG did not affect the total number of COX-2expressing cells in the intestine; however, in response to LGG, the number of COX-2-expressing cells associated with the crypts increased.

In the normal colon, there is a population of cells in the lamina propria near the upper crypts that constitutively express COX-2. ${ }^{4}$ These cells also express CD44, CD29, CD105 and CD106, a pattern consistent with MSCs. ${ }^{31}$ In DSS-induced colitis, there are COX-2-dependent protective effects that preserve epithelial proliferation, even though DSS-colitis is not associated with increased COX-2 expression or increased $\mathrm{PGE}_{2}$ production. In the DSS model, COX-2-expressing MSCs migrated from near the upper crypt epithelium to near the lower crypt epithelial cells. This process is MyD88 dependent. Our interpretation of these findings was that the migration of COX2-expressing cells allowed a high concentration of $\mathrm{PGE}_{2}$ in the area immediately surrounding the proliferating crypt cells and that $\mathrm{PGE}_{2}$ supported epithelial cell proliferation. In the present study, we observed a similar pattern now in the small intestine. The small intestinal MSCs that constitutively express high levels of COX-2 migrated in response to LGG in a TLR-2dependent fashion. The migration of these COX-2-positive cells was associated with diminished epithelial apoptosis and increased crypt survival in response to radiation injury.

These migrating cells express COX-2 and surface proteins associated with MSCs. The constitutive expression of high levels of COX-2 is specific to intestinal and colonic MSCs, as bone marrow-derived MSCs expressed little COX-2 (figure 6B). The high level of COX-2 expression in colonic MSCs is not TLR dependent, but dependent on fibroblast growth factor 9 (FGF9) produced by epithelial cells. ${ }^{31}$ In the present study, the migration of these intestinal MSCs was found to be TLR-2 specific. How TLR-2 signalling is involved in their migration is not clear. It is possible that the TLR-2 signalling occurs in the MSCs, as these cells functionally express the gene; however, it also possible that the TLR-2 ligand binds to a cell in or near the crypt, and this cell produces a chemotactic factor that promotes the migration of the MSCs towards the crypt. $\mathrm{PGE}_{2}$ produced by these COX-2expressing MSCs would act to protect adjacent epithelial stem

\section{(Continued)}

COX-2 quantitative reverse transcription-PCR of small intestinal (SI) and colonic (C) MSCs relative to bone marrow-derived (BM) MSCs. (C) Flow cytometry for cell surface markers of isolated COX-2-expressing MSCs. Gating based on isotype control. (D) mRNA expression of murine TLR ligands relative to actin $(\log 10)$ in isolated SI-MSCs. (E) Tumour necrosis factor (TNF) $\alpha$ and COX-2 mRNA expression in MSCs after stimulation with a TLR-2 ligand (Pam3CSK4) for $18 \mathrm{~h}$. FSC, forward scatter ${ }^{*} p<0.05,{ }^{* *} p<0.01$ vs the control (CTRL). 
cells from radiation-induced apoptosis. The close physical proximity of the $\mathrm{PGE}_{2}$-producing MSCs and the epithelial stem cells is important because $\mathrm{PGE}_{2}$ is unstable and therefore only acts over short distances. We have now demonstrated TLRdependent migration of COX-2-positive MSCs as being involved in DSS-colitis and radioprotective in the small intestine. This raises the possibility that TLR-dependent migration of COX-2expressing MSCs may be a generalised mechanism for epithelial protection in the intestine and colon.

These studies were carried out using 12 Gy of total body irradiation, with epithelial apoptosis, crypt survival, weight loss and duration of survival used as biological end points. This is the standard protocol for intestinal radioprotection studies in the mouse. As no animal model exactly mirrors the human disease, certain distinctions should be noted. Humans undergoing radiation therapy for abdominal malignancy receive lower, fractionated doses of radiation to the intestine. The major clinical effects in humans-diarrhoea and vomiting-typically develop during the second week of radiotherapy (cumulative radiation dose of 15-20 Gy). ${ }^{3}$ Mice do not typically develop diarrhoea or vomiting in response to radiation but exhibit weight loss and, if the enterocyte depletion is high, death over the first 10 days. Nonetheless, this study has relevance for radioprotection in patients in that radiation-induced diarrhoea is a product of radiation-induced epithelial apoptosis in the intestine, and other agents that reduce radiation-induced apoptosis are also effective radioprotective agents. ${ }^{11} 45$ Furthermore, agents demonstrated to be radioprotective in mice using this radiation protocol are also radioprotective in primates. ${ }^{11}$

This study raises the possibility of using probiotics as radioprotective agents in man and provides insight into the administrative route, timing and mechanism of probioticmediated intestinal radioprotection. As radioprotectants, probiotics have the advantage of providing wide luminal coverage and act locally at the mucosal surface to protect the normal epithelium without having a systemic distribution. Although probiotics such as LGG $^{46}$ have an excellent human safety profile, if viable bacteria were used in human trials, close monitoring would be critical. Further studies directed at delineating mechanisms and optimising the radioprotective effects of probiotics are indicated.

Funding This work was supported in part by the National Institutes of Health grants DK55753 and DK33165 (to WFS), DK089016 and L30 RR030244 (to MAC), DK071619 and DK07161-90251 (to TSS) and P30-DK52574 (to the Washington University Digestive Diseases Research Core). This work was also supported in part by the Crohn's and Colitis Foundation of America and a Global Probiotics Council Young Investigator Award (both to MAC). We thank Lynne Foster and Ellen Bettonville for additional technical assistance, and Dr Parag Parikh for useful discussions on this work.

\section{Competing interests None.}

Contributors Study concept and design: MAC, WFS. Acquisition of data: MAC, MSR, TER, XE, CM, MSW, JMM, GMN. Analysis and interpretation of data: MAC, WFS, TER, CM, TSS, XE, GMN. Drafting of the manuscript: MAC, WFS. Critical revision of the manuscript for important intellectual content: TSS, TER, MSR, MSW, CM, XE. Statistical analysis: MAC. Obtained funding: MAC, WFS, TSS. Approved final version of manuscript: all authors.

Provenance and peer review Not commissioned; externally peer reviewed.

Data sharing statement We are happy to share our data. All the reagents used are commercially available.

\section{REFERENCES}

1. Williams JP, Brown SL, Georges GE, et al. Animal models for medical countermeasures to radiation exposure. Radiat Res 2010;173:557-78.

2. Potten CS. A comprehensive study of the radiobiological response of the murine (BDF1) small intestine. Int J Radiat Biol 1990;58:925-73.
3. Bismar MM, Sinicrope FA. Radiation enteritis. Curr Gastroenterol Rep 2002:4:361-5.

4. Brown SL, Riehl TE, Walker MR, et al. Myd88-dependent positioning of Ptgs2expressing stromal cells maintains colonic epithelial proliferation during injury. J Clin Invest 2007:117:258-69.

5. Fukata M, Michelsen KS, Eri R, et al. Toll-like receptor-4 is required for intestinal response to epithelial injury and limiting bacterial translocation in a murine model of acute colitis. Am J Physiol Gastrointest Liver Physiol 2005;288:G1055-65.

6. Rakoff-Nahoum S, Paglino J, Eslami-Varzaneh F, et al. Recognition of commensal microflora by toll-like receptors is required for intestinal homeostasis. Cell 2004;118:229-41.

7. Stenson WF. Toll-like receptors and intestinal epithelial repair. Curr Opin Gastroenterol 2008;24:103-7.

8. Packey CD, Ciorba MA. Microbial influences on the small intestinal response to radiation injury. Curr Opin Gastroenterol 2010;26:88-94.

9. Crawford PA, Gordon JI. Microbial regulation of intestinal radiosensitivity. Proc Nat Acad Sci U S A 2005;102:13254-9.

10. Riehl T, Cohn S, Tessner T, et al. Lipopolysaccharide is radioprotective in the mouse intestine through a prostaglandin-mediated mechanism. Gastroenterology 2000:118:1106-16.

11. Burdelya LG, Krivokrysenko VI, Tallant TC, et al. An agonist of toll-like receptor 5 has radioprotective activity in mouse and primate models. Science 2008:320:226-30.

12. Vijay-Kumar M, Aitken JD, Sanders CJ, et al. Flagellin treatment protects agains chemicals, bacteria, viruses, and radiation. J Immunol 2008;180:8280-5.

13. Jones RM, Sloane VM, Wu H, et al. Flagellin administration protects gut mucosal tissue from irradiation-induced apoptosis via MKP-7 activity. Gut 2011:60:648-57

14. Williams MD, Ha CY, Ciorba MA. Probiotics as therapy in gastroenterology: a study of physician opinions and recommendations. J Clin Gastroenterol 2010;44:631-6.

15. Floch MH, Walker WA, Guandalini S, et al. Recommendations for probiotic use-2008. J Clin Gastroenterol 2008;42(Suppl 2):S104-8.

16. Sartor RB. Microbial influences in inflammatory bowel diseases. Gastroenterology 2008:134:577-94.

17. Yan F, Cao H, Cover TL, et al. Soluble proteins produced by probiotic bacteria regulate intestinal epithelial cell survival and growth. Gastroenterology 2007:132:562-75.

18. Lin PW, Myers LE, Ray L, et al. Lactobacillus rhamnosus blocks inflammatory signalling in vivo via reactive oxygen species generation. Free Radic Biol Med 2009;47:1205-11.

19. Ciorba MA, Stenson WF. Probiotic therapy in radiation-induced intestinal injury and repair. Ann N Y Acad Sci 2009;1165:190-4.

20. Demirer S, Aydintug S, Aslim B, et al. Effects of probiotics on radiation-induced intestinal injury in rats. Nutrition 2006;22:179-86.

21. Seal M, Naito $Y$, Barreto $R$, et al. Experimental radiotherapy-induced enteritis: a probiotic interventional study. J Dig Dis 2007;8:143-7.

22. Dong MY, Chang TW, Gorbach SL. Effects of feeding lactobacillus GG on lethal irradiation in mice. Diagn Microbiol Infect Dis 1987;7:1-7.

23. Delia $\mathbf{P}$, Sansotta G, Donato $V$, et al. Use of probiotics for prevention of radiationinduced diarrhea. World J Gastroenterol 2007:13:912-15.

24. Giralt J, Regadera JP, Verges R, et al. Effects of probiotic Lactobacillus Case DN-114 001 in prevention of radiation-induced diarrhea: results from multicenter, randomized, placebo-controlled nutritional trial. Int J Radiat Oncol Biol Phys 2008;71:1213-19.

25. Urbancsek H, Kazar T, Mezes I, et al. Results of a double-blind, randomized study to evaluate the efficacy and safety of antibiophilus in patients with radiation-induced diarrhoea. Eur J Gastroenterol Hepatol 2001;13:391-6.

26. Chitapanarux I, Chitapanarux T, Traisathit P, et al. Randomized controlled trial of live lactobacillus acidophilus plus Bifidobacterium bifidum in prophylaxis of diarrhea during radiotherapy in cervical cancer patients. Radiat Oncol 2010;5:31.

27. Pritchard DM, Potten CS, Korsmeyer SJ, et al. Damage-induced apoptosis in intestinal epithelia from bcl-2-null and bax-null mice: investigations of the mechanistic determinants of epithelial apoptosis in vivo. Oncogene 1999;18:7287-93.

28. Potten CS, Merritt A, Hickman J, et al. Characterization of radiation-induced apoptosis in the small intestine and its biological implications. Int J Radiat Biol 1994;65:71-8

29. Miettinen M, Vuopio-Varkila J, Varkila K. Production of human tumor necrosis facto alpha, interleukin-6, and interleukin-10 is induced by lactic acid bacteria. Infect Immun 1996;64:5403-5

30. Yan F, Polk DB. Probiotic bacterium prevents cytokine-induced apoptosis in intestinal epithelial cells. J Biol Chem 2002;277:50959-65.

31. Walker MR, Brown SL, Riehl TE, et al. Growth factor regulation of prostaglandinendoperoxide synthase 2 (Ptgs2) expression in colonic mesenchymal stem cells. J Biol Chem 2010:285:5026-39.

32. Ciorba MA, Bettonville EE, McDonald KG, et al. Induction of IDO-1 by immunostimulatory DNA limits severity of experimental colitis. J Immunol 2010;184:3907-16.

33. Zheng L, Riehl TE, Stenson WF. Regulation of colonic epithelial repair in mice by toll-like receptors and hyaluronic acid. Gastroenterology 2009;137:2041-51.

34. Nava GM, Friedrichsen HJ, Stappenbeck TS. Spatial organization of intestinal microbiota in the mouse ascending colon. ISME J 2011;5:627-38. 
35. Case RJ, Boucher $Y$, Dahllof I, et al. Use of $16 \mathrm{~S}$ rRNA and rpoB genes as molecular markers for microbial ecology studies. Appl Environ Microbiol 2007;73:278-88.

36. Brandt K, Alatossava T. Specific identification of certain probiotic Lactobacillus rhamnosus strains with PCR primers based on phage-related sequences. Int $J$ Food Microbiol 2003;84:189-96.

37. Dommels YE, Kemperman RA, Zebregs YE, et al. Survival of Lactobacillus reuteri DSM 17938 and Lactobacillus rhamnosus GG in the human gastrointestinal tract with daily consumption of a low-fat probiotic spread. Appl Environ Microbiol 2009;75:6198-204.

38. Scoville DH, Sato T, He XC, et al. Current view: intestinal stem cells and signaling. Gastroenterology 2008;134:849-64.

39. Rachmilewitz D, Katakura K, Karmeli F, et al. Toll-like receptor 9 signaling mediates the anti-inflammatory effects of probiotics in murine experimental colitis. Gastroenterology 2004;126:520-8.

40. Tao Y, Drabik KA, Waypa TS, et al. Soluble factors from Lactobacillus GG activate MAPKs and induce cytoprotective heat shock proteins in intestinal epithelial cells. Am J Physiol Cell Physiol 2006;290:C1018-30.
41. Velez MP, Petrova Ml, Lebeer $\mathrm{S}$, et al. Characterization of MabA a modulator of Lactobacillus rhamnosus GG adhesion and biofilm formation. FEMS Immunol Med Microbiol 2010;59:386-98.

42. Tuomola EM, Salminen SJ. Adhesion of some probiotic and dairy Lactobacillus strains to Caco-2 cell cultures. Int J Food Microbiol 1998;41:45-51.

43. Tuomola EM, Ouwehand AC, Salminen SJ. The effect of probiotic bacteria on the adhesion of pathogens to human intestinal mucus. FEMS Immunol Med Microbiol 1999;26:137-42

44. O'Mahony L, McCarthy J, Kelly P. et al. Lactobacillus and bifidobacterium in irritable bowel syndrome: symptom responses and relationship to cytokine profiles. Gastroenterology 2005;128:541-51.

45. Yamamoto T, Kinoshita M, Shinomiya N, et al. Pretreatment with ascorbic acid prevents lethal gastrointestinal syndrome in mice receiving a massive amount of radiation. J Radiat Res (Tokyo) 2010;51:145-56.

46. Snydman DR. The safety of probiotics. Clin Infect Dis 2008;46(Suppl 2):S104-111; discussion S144-51.

\section{Have confidence in your decision making.}
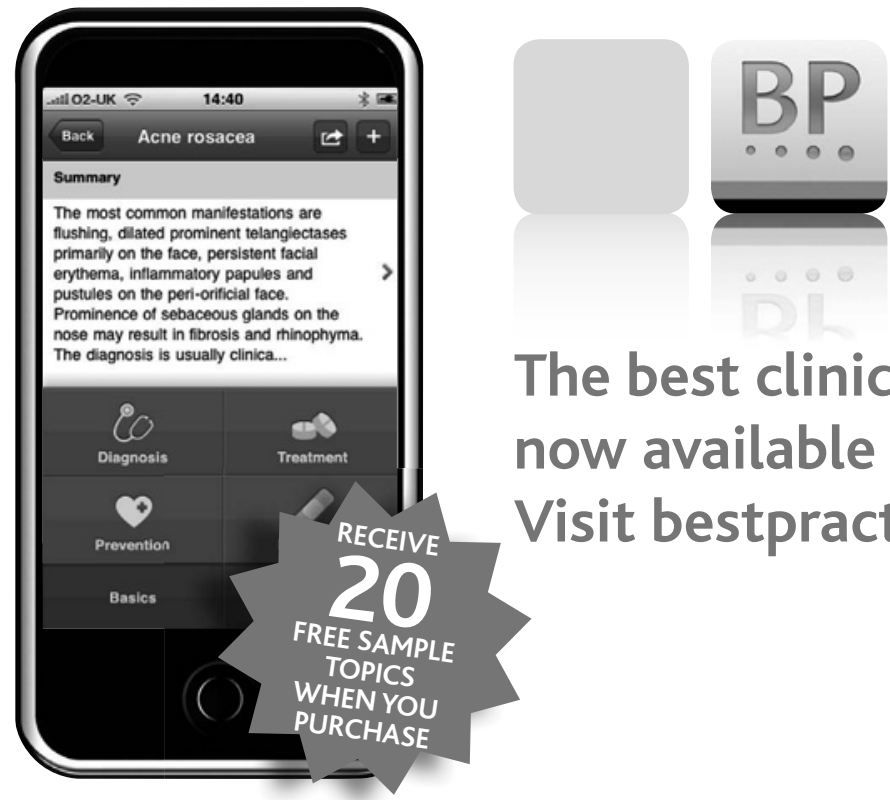

The best clinical decision support tool is now available as an app for your iPhone. Visit bestpractice.bmj.com/app

\section{clinicians $\bullet$ medical students $\bullet$ nurses $\bullet$ healthcare practitioners}

